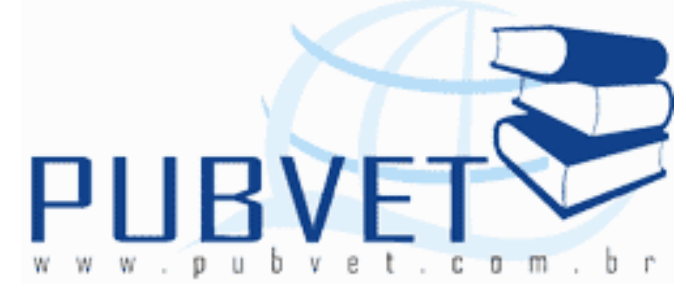

PUBVET, Publicações em Medicina Veterinária e Zootecnia.

\title{
Consumo voluntário de volumosos estimado por meio de parâmetros de degradação ruminal
}

Adriana de Souza Martins ${ }^{1}$, Telma Teresinha Berchielli ${ }^{2}$, Ana Karina Dias

Salman ${ }^{3}$, João Paulo Guimarães Soares ${ }^{4}$, Meiby Carneiro de Paula Leite ${ }^{5}$

${ }^{1}$ Docente do Departamento de Zootecnia da Universidade Estadual de Ponta Grossa - UEPG.

${ }^{2}$ Docente da Faculdade de Ciências Agrárias e Veterinárias - FCAV - UNESP Jaboticabal

${ }^{3}$ Pesquisadora da Embrapa Rondônia.

${ }^{4}$ Pesquisador da Embrapa Cerrados.

${ }^{5}$ Docente da Universidade Federal do Recôncavo da Bahia - UFRB

\section{Resumo}

O objetivo do presente estudo foi de estimar o consumo voluntário de matéria seca (CMS) através de equações baseadas nos parâmetros de degradação ruminal da silagem de milho, do feno de Tifton-85 cortado aos 30 e aos 90 dias, do bagaço de cana e da palha de arroz. Foram utilizados seis bovinos mestiços, com peso vivo médio de $440 \mathrm{~kg}$, fistulados no rúmen. A degradabilidade foi determinada através da técnica in situ, utilizando-se sacos de náilon no rúmen, incubados nos seguintes tempos: 3, 6, 12, 24, 48, 72 e 96 horas. A partir dos parâmetros de degradação, foram estimados os 
consumos pelas seguintes equações de predição: 1) CMS = -1,19+0,035 (a+ b) $+28,5 c$; 2) CMS $=-0,822+0,0748(a+b)+40,7 c ; 3)$ CMS $=-8,286+$ $0,266 a+0,102 b+17,696 c$ e 4) CMS $=$ [\%FDN na MS]*[consumo de FDN ] / $[(1-a-b) / k p+b /(c+k p)] / 24]$. As estimativas de CMS dos volumosos de baixa qualidade (Feno 90 e bagaço de cana) foram subestimadas e superestimadas, respectivamente, pelas equações 3 e 2 . As estimativas de CMS da silagem de milho pelas equações 3 e 4 (6,31 e 9,04 kg MS/dia, respectivamente) foram maiores em relação as estimativas para os demais volumosos. Verificou-se, para todas as equações, menor consumo do bagaço de cana em relação aos demais volumosos. Houve alta correlação entre a taxa de degradação e o enchimento ruminal com as equações de consumo. Os parâmetros de degradação ruminal podem ser utilizados na estimativa do consumo de MS de volumosos, no entanto, considerando as equações avaliadas nesse estudo, a acurácia da estimativa é dependente das características nutricionais dos volumosos.

Palavras-chave: bovinos, degradabilidade, enchimento ruminal, taxa de passagem

\title{
Voluntary intake of roughage estimated by rumen degradation parameters
}

\begin{abstract}
This study aimed to estimate the dry matter intake (DMI) by ruminal degradation parameters of corn silage, Tifton-85 hay cut at 30 and 90 days, sugarcane bagasse and rice straw. Six crossbred cattle rumen fistulated with average live weight of $340 \mathrm{~kg}$ were used. The degradability was determined by in situ technique using nylon bags rumen incubated at the following periods: 3, $6,12,24,48,72$ and 96 hours. By parameters of degradation, the intake was predicted using the following equations: 1$)$ DMI $=-1.19+0.035(a+b)+$ $28.5 c ; 2)$ DMI $=-0.822+0.0748(a+b)+40.7 c ; 3) D M I=-8.286+0.266 a+$ $0.102 b+17.696 c$ and 4$) D M I=[\% N D F$ in the DM] $*[N D F$ intake $] /[(1-a-b) / k p$
\end{abstract}


$+b /(c+k p)] / 24]$. ]. The DMI of low quality roughages (90-day hay and bagasse) predicted by 3 and 2 equations were underestimated and overestimated, respectively. The prediction of corn silage DMI by 3 and 4 equations (6.31 and $9.04 \mathrm{~kg} \mathrm{DM} /$ day, respectively) were higher than the other evaluated roughages. For all equations, the DMI of bagasse was lower than the other roughages. There was a high correlation between degradation rate and rumen fill with prediction equations of voluntary intake. Parameters of rumen degradability can be used for prediction of roughage DMI, but considering the equations evaluated in this study, the prediction accuracy depends on the nutritional traits of roughage.

Keywords: cattle, degradability, passage rate, rumen fill

\section{Introdução}

A ingestão de matéria seca é de fundamental importância na nutrição animal, pois determina a quantidade de nutrientes disponíveis ao animal para sua saúde e produção. A acurácia na estimativa do consumo auxilia na formulação de dietas de modo a prevenir desperdícios ou mesmo a deficiência de nutrientes ao organismo, promovendo seu uso de forma eficiente.

Em ruminantes, fatores fisiológicos, físicos e psicogênicos parecem controlar o consumo. Os fatores físicos predominam em dietas de baixa qualidade, em que o consumo é limitado pelo volume ocupado pela dieta e pela capacidade anatômica do rúmen-retículo. Os moduladores psicogênicos referem-se à resposta do animal a fatores estimuladores ou inibidores do alimento ou do ambiente de alimentação, os quais não estão relacionados à concentração de energia do alimento ou à repleção ruminal (Mertens, 1994).

Grande parte das equações de predição do consumo testadas na literatura baseia-se no princípio de que o consumo de MS pelos animais é definido pelo atendimento dos requerimentos de energia ou também são desenvolvidas por regressão, incluindo diferentes fatores que interferem no consumo de MS 
(NRC, 2001). Entretanto, a validação destas equações para a estimativa do consumo ainda é um objetivo a ser alcançado, uma vez que na maioria dos trabalhos, os resultados são ainda inconsistentes.

Ainda com relação ao consumo de matéria seca, o reflexo da relação volumoso:concentrado determina que podem ocorrer diferentes efeitos (Moore, 1980). Esses são descritos como associativos, podendo ser classificados como substitutivos, aditivos ou combinados. Segundo o autor, no efeito substitutivo, o consumo de energia proveniente do concentrado se mantém constante, e o da forragem decresce. Para o efeito aditivo ocorre o inverso, o consumo de concentrado aumenta e o de forragem se estabiliza. Por último, no efeito combinado, observa-se que o consumo de concentrado aumenta e o consumo da forragem diminui.

É reconhecido que fatores relacionados ao animal, à dieta e ao ambiente são fundamentais na determinação do consumo, porém, é preciso determinar o efeito das variáveis que compõem cada fator sobre as estimativas de consumo, permitindo que em estudos futuros, equações mais precisas e ajustadas aos modelos sejam formuladas e validadas.

O objetivo do presente estudo foi estimar o consumo voluntário por meio de equações baseadas nos parâmetros de degradação ruminal de alguns volumosos utilizados na alimentação de bovinos.

\section{Material e Métodos}

O trabalho foi desenvolvido no Setor de Avaliação de Alimentos do Departamento de Zootecnia da Faculdade de Ciências Agrárias e Veterinárias Campus de Jaboticabal/UNESP-SP.

O ensaio de degradabilidade in situ foi realizado utilizando-se seis bovinos, machos mestiços (Holandês x Zebu), com peso vivo médio de $440 \mathrm{~kg}$, providos de cânula no rúmen, mantidos em baias individuais, cobertas, com comedouro e bebedouro. A ração total fornecida aos animais foi formulada baseando-se na 
ingestão de energia metabolizável fermentável e de proteína degradável no rúmen, segundo as recomendações do AFRC (1993). A proporção volumoso concentrado foi de 70:30, sendo o concentrado composto de milho e levedura e o volumoso de silagem de milho, bagaço de cana, feno de Tifton cortado aos 30 e aos 90 dias e palha de arroz. A ração total (volumoso + concentrado) foi fornecida duas vezes ao dia, às 8:00 h e às 15:00 $\mathrm{h}$, permitindo-se sobras de aproximadamente $10 \%$ do oferecido.

A composição química dos ingredientes da dieta encontra-se na tabela 1. A composição percentual (\% MS) dos ingredientes da dieta foi de 11,7\%; $18,3 \%$ e $14 \%$, respectivamente, para o milho, levedura e para cada volumoso que constituiu a dieta (silagem de milho, feno de Tifton cortado aos 30 e aos 90 dias, palha de arroz e bagaço de cana). Os teores de MS, PB, FDN e FDA da dieta foram 83,$10 ; 12,94 ; 49,96$ e $29,53 \%$, respectivamente.

Tabela 1. Composição química dos ingredientes da dieta (\% MS).

\begin{tabular}{lccccc}
\hline Ingredientes & MS & PB & FDN & FDA & NDT $^{3}$ \\
\hline Silagem de milho & 32,27 & 7,74 & 46,28 & 24,32 & 65 \\
Bagaço de cana & 45,32 & 1,21 & 85,12 & 64,72 & 51 \\
Feno de Tifton30 & 94,24 & 8,75 & 69,96 & 34,58 & 62 \\
Feno de Tifton $90^{2}$ & 92,00 & 5,55 & 79,76 & 42,99 & 54 \\
Palha de arroz & 92,22 & 6,52 & 67,20 & 40,63 & 56 \\
Milho & 90,32 & 12,00 & 12,02 & 5,16 & 87 \\
Levedura & 89,08 & 37,80 & - & - & 83 \\
\hline
\end{tabular}

${ }^{1}$ Colhido aos 30 dias de rebrote. ${ }^{2}$ Colhido aos 90 dias de rebrote. ${ }^{3}$ Calculado segundo NRC (2001): \%NDT $=\mathrm{PB} d+2,25 \mathrm{EE} d+\mathrm{FDN} d+\mathrm{CNF} d$, em que $d$ equivale ao respectivo nutriente digerível e $\mathrm{CNF}=$ carboidratos não fibrosos.

Aproximadamente $5 \mathrm{~g}$ de cada um dos volumosos avaliados (silagem de milho, bagaço de cana-de-açúcar, feno de Tifton-85 cortado aos 30 dias e aos 90 dias e palhada de arroz) foram moídos a $5 \mathrm{~mm}$ e acondicionadas em bolsas de náilon ( $7 \times 14 \mathrm{~cm}, 50 \mu \mathrm{m}$ de tamanho de poro), as quais foram incubadas, em duplicata, nos seguintes tempos: $0 ; 3 ; 6 ; 12 ; 24 ; 48 ; 72$ e 96 horas. Os 
resíduos foram analisados para matéria seca (MS), fibra em detergente neutro (FDN) e fibra em detergente ácido (FDA), de acordo com as metodologias descritas por Silva \& Queiroz (2002). O teor de nutrientes digestíveis totais (NDT) foi calculado segundo a equação: \%NDT $=\mathrm{PB} d+2,25 \mathrm{EE} d+\mathrm{FDN} d+$ CNF $d$, em que $d$ equivale ao respectivo nutriente digerível; e os carboidratos não fibrosos (CNF) foram calculados pela fórmula: CNF $=100-(P B+F D N c p$ $+\mathrm{MM}+\mathrm{EE})(\mathrm{NRC}, 2001)$, em que: $\mathrm{FDNcp}=$ fibra em detergente neutro corrigida para proteína.

Os dados sobre o desaparecimento da MS e da FDN dos volumosos foram ajustados ao modelo proposto por Mehrez \& Ørskov (1977) expresso por: $p=$ $a+b\left(1-e^{-c t}\right)$ em que: $p=$ quantidade de nutriente degradado no tempo $t ; a$ = fração solúvel em água, $b$ = fração insolúvel em água, mas potencialmente degradável; $c$ = taxa de degradação da fração $b$ e $t$, tempo de incubação em horas. A fração solúvel foi obtida incubando-se o tempo 0 em água a $39^{\circ} \mathrm{C}$ durante uma hora. A degradabilidade efetiva (DE) foi calculada segundo o modelo proposto por ØRSKOV e McDonald (1979): DE $=a+\left[\left(b \cdot k_{d}\right) /\left(k_{d}+\right.\right.$ $\left.\mathrm{k}_{\mathrm{p}}\right)$ ], em que: $\mathrm{k}_{\mathrm{p}}$ equivale à taxa estimada de passagem de sólidos no rúmen por hora, levando-se em consideração a taxa de passagem de sólidos no rúmen de $2 \%$ /hora, atribuída a um consumo alimentar baixo (ARC, 1984) e $k_{d}$ $a$ taxa de degradação da fração $b$.

$\mathrm{Na}$ tabela 2 são apresentados os parâmetros de degradação dos volumosos avaliados.

A partir dos parâmetros de degradação ruminal foram estimados os consumos de matéria seca (CMS) por meio das seguintes equações: Von Keyserlingk \& Mathison (1989) em que CMS $=-1,19+0,035(a+b)+28,5 c$ (1); Ørskov et al. (1988) em que CMS $=-0,822+0,0748(a+b)+40,7 c$ (2); Shem et al. (1995) na qual CMS $=-8,286+0,266 a+0,102 b+17,696 c$ (3) e Madsen et al. (1997) em que CMS $=(1 /$ FDN $) *$ CPFDN (4), onde CPFDN = consumo potencial de FDN ( $\mathrm{kg} / \mathrm{dia})$. 
Tabela 2. Fração solúvel (a), potencialmente degradável (b), taxa de degradação (c) e a degradabilidade efetiva (DE) da matéria seca dos volumosos.

\begin{tabular}{lcccc}
\hline Ingredientes & $\mathrm{a}(\%)$ & $\mathrm{b}(\%)$ & $\mathrm{c}(\% / \mathrm{h})$ & $\mathrm{DE}(\%)^{3}$ \\
\hline Silagem de milho & 35,09 & 45,95 & 3,23 & 62,60 \\
Bagaço de cana & 4,94 & 37,92 & 4,05 & 29,82 \\
Feno de Tifton30 $30^{1}$ & 22,37 & 54,36 & 5,83 & 62,68 \\
Feno de Tifton $90^{2}$ & 13,72 & 41,15 & 4,32 & 41,48 \\
Palha de arroz & 18,48 & 51,44 & 5,03 & 55,30 \\
\hline
\end{tabular}

${ }^{1}$ Colhido aos 30 dias de rebrote. ${ }^{2}$ Colhido aos 90 dias de rebrote. ${ }^{3}$ Considerando taxa de passagem de $2 \% / h$.

Na equação proposta por Madsen et al. (1997), o cálculo do CPFDN leva em consideração a capacidade ruminal (CR) e o enchimento físico ruminal $(E R)$, sendo obtido pela fórmula: CPFDN ( $\mathrm{kg} / \mathrm{dia})=\mathrm{CR} / \mathrm{ER}$ (dias). A estimativa do enchimento físico ruminal (ER) é obtida pela seguinte equação: ER (dias) = $(1-a-b) / k+(b / c+k) / 24$. Estes dados referem-se aos parâmetros de degradação da FDN do volumoso. Para o cálculo do consumo utilizando este modelo foram consideradas três taxas de passagem $(\mathrm{k}): 2 \% / \mathrm{h}, 3 \% / \mathrm{h}$ e $5 \% / \mathrm{h}$. No presente estudo, a capacidade ruminal foi de 4,83 $\mathrm{kg}$ de FDN, obtida por meio do cálculo de $1,1 \%$ do peso corporal, proposto por Madsen et al. (1997). De acordo com os autores, a estimativa do material não degradado presente no rúmen no tempo $t$ é obtida por meio da fração remanescente na bolsa de náilon, a qual ainda não saiu do rúmen-retículo. Assumindo-se que o alimento permaneça no rúmen até que seja degradado, o enchimento ruminal (ER), representado por esta fração no rúmen, é calculado pela equação acima, em um período de $24 \mathrm{~h}$.

Utilizou-se o delineamento inteiramente casualizado, com seis repetições (animais), em esquema fatorial $5 \times 4$ (cinco volumosos e quatro equações). A análise de variância e a comparação de médias pelo teste de Tukey, em nível de $5 \%$ de significância, foram obtidas pelo PROC GLM DO SAS (SAS Institute, 
2000). As correlações de Pearson dos parâmetros de degradação com as respectivas equações de predição foram analisadas pelo programa SAS (2000).

\section{Resultados e Discussão}

Nas tabelas 3 e 4 são apresentadas as médias do consumo de matéria seca dos volumosos estimadas pelas diferentes equações e expressas em $\mathrm{kg}$ MS/dia e \% do Peso Vivo (PV), respectivamente.

Tabela 3. Médias do consumo de matéria seca ( $\mathrm{kg} \mathrm{MS/dia)} \mathrm{dos} \mathrm{volumosos}$ estimadas pelas diferentes equações.

\begin{tabular}{lcccccc}
\hline Equação & $\begin{array}{c}\text { Silagem de } \\
\text { milho }\end{array}$ & Feno $30^{1}$ & Feno $90^{2}$ & $\begin{array}{c}\text { Bagaço de } \\
\text { cana }\end{array}$ & $\begin{array}{c}\text { Palha de } \\
\text { arroz }\end{array}$ & C.V. \\
\hline V. Keyserlingk $^{3}$ & $2,57 \mathrm{Be}$ & 3,16Ad & $1,96 \mathrm{Cd}$ & $1,46 \mathrm{Cd}$ & $2,70 \mathrm{ABd}$ & 12,4 \\
Ørskov $^{4}$ & $6,55 \mathrm{ABd}$ & $7,29 \mathrm{Ac}$ & $5,04 \mathrm{Cc}$ & $4,03 \mathrm{Dc}$ & $6,48 \mathrm{Bc}$ & 7,4 \\
Shem $^{5}$ & $6,31 \mathrm{Ad}$ & $4,24 \mathrm{Bd}$ & $0,41 \mathrm{De}$ & $-2,39 \mathrm{Ee}$ & $2,80 \mathrm{Cd}$ & 6,5 \\
Madsen $^{6}$ & $9,04 \mathrm{Ac}$ & $8,06 \mathrm{Bc}$ & $5,00 \mathrm{Dc}$ & $4,03 \mathrm{Ec}$ & $6,59 \mathrm{Cc}$ & 8,1 \\
Madsen $^{7}$ & $12,31 \mathrm{Ab}$ & $10,74 \mathrm{Bb}$ & $6,99 \mathrm{Db}$ & $5,78 \mathrm{~Eb}$ & $9,06 \mathrm{Cb}$ & 7,7 \\
Madsen $^{8}$ & $18,42 \mathrm{Aa}$ & $15,14 \mathrm{Ba}$ & $10,69 \mathrm{Da}$ & $9,13 \mathrm{Ea}$ & $13,55 \mathrm{Ca}$ & 6,3 \\
C.V. & 9,31 & 6,86 & 5,13 & 4,24 & 5,45 & \\
\hline
\end{tabular}

${ }^{1}$ Colhido aos 30 dias de rebrote. ${ }^{2}$ Colhido aos 90 dias de rebrote. ${ }^{3}$ VON KEYSERLINGK e MATHISON (1989). ${ }^{4} \varnothing$ RSKOV et al. (1988). ${ }^{5}$ SHEM et al. (1995). $6,7,8$ MADSEN et al. (1997). Consumo estimado considerando taxa de passagem de $2 \% / \mathrm{h}, 3 \% / \mathrm{h}$ e $5 \% / \mathrm{h}$, respectivamente. Médias seguidas de letras diferentes, minúscula na coluna e maiúscula na linha, diferem $(P<0,05)$ pelo teste de Tukey. $C . V .=$ coeficiente de variação.

As equações de predição apresentaram coeficientes de determinação $\left(R^{2}\right)$ elevados, sendo os valores de 0,82, 0,88, 0,93 e 0,96 para as equações de Von Keyserlingk \& Mathison (1989), Ørskov et al. (1988), Shem et al. (1995) e Madsen et al. (1997), respectivamente.

As equações de Shem et al. (1995) e Madsen et al. (1997) (considerando taxa de passagem de $2 \% / \mathrm{h}$ ) indicaram maior consumo de silagem de milho $(P<0,05)$ em relação aos demais volumosos. Porém, as equações de Ørskov et al. (1988) e Von Keyserlingk \& Mathison (1989) revelaram maior consumo do feno de Tifton 30, seguido pela palha de arroz e pela silagem de milho, 
respectivamente. De acordo com as referidas equações, os volumosos de melhor qualidade (silagem de milho e feno colhido com 30 dias), ou seja, com maior DE da MS, teor de PB e de energia e menor teor de FDN, proporcionaram os maiores consumos quando comparados aos volumosos de baixa qualidade (feno colhido com 90 dias e bagaço de cana).

Tabela 4. Consumo de matéria seca dos volumosos, expresso em percentagem do peso vivo (\%PV), estimado pelas equações de predição.

\begin{tabular}{|c|c|c|c|c|c|}
\hline Equação & $\begin{array}{l}\text { Silagem de } \\
\text { milho }\end{array}$ & Feno $30^{1}$ & Feno $90^{2}$ & $\begin{array}{c}\text { Bagaço de } \\
\text { cana }\end{array}$ & $\begin{array}{c}\text { Palha de } \\
\text { arroz }\end{array}$ \\
\hline V. Keyserlingk ${ }^{3}$ & 0,58 & 0,72 & 0,45 & 0,33 & 0,61 \\
\hline Ørskov 4 & 1,49 & 1,66 & 1,15 & 0,92 & 1,47 \\
\hline Shem $^{5}$ & 1,43 & 0,96 & 0,09 & - & 0,64 \\
\hline Madsen 6 & 2,05 & 1,83 & 1,14 & 0,92 & 1,50 \\
\hline Madsen $^{7}$ & 2,80 & 2,44 & 1,59 & 1,31 & 2,06 \\
\hline Madsen ${ }^{8}$ & 4,19 & 3,44 & 2,42 & 2,01 & 3,08 \\
\hline $\begin{array}{l}\text { Dados } \\
\text { literatura }\end{array}$ & $1,8^{9}$ & $1,7^{10}$ & $1,3^{11}$ & $0,7^{12}$ & $1,6^{13}$ \\
\hline
\end{tabular}

${ }^{1}$ Colhido aos 30 dias de rebrote. ${ }^{2}$ Colhido aos 90 dias de rebrote. ${ }^{3}$ VON KEYSERLINGK e MATHISON (1989). ${ }^{4} \varnothing$ RSKOV et al. (1988). ${ }^{5}$ SHEM et al. (1995). $6,7,8$ MADSEN et al. (1997). Consumo estimado considerando taxa de passagem de $2 \% / \mathrm{h}, 3 \% / \mathrm{h}$ e $5 \% / \mathrm{h}$, respectivamente. ${ }^{9}$ CABRAL et al. (2006); 10,11 MENDES NETO et al. (2007); ${ }^{12}$ PIRES et al. (2004); ${ }^{13}$ MGHENI et al. (2001).

O consumo de alimentos de baixa qualidade é limitado por aspectos físicos, definidos pelo volume ocupado no rúmen retículo e pela capacidade anatômica destes compartimentos, de forma que os animais dificilmente ingerem energia suficiente para atender seus requerimentos nutricionais. Isto geralmente ocorre com animais em pastejo (Cabral et al., 2006) ou consumindo volumosos de baixa qualidade. Segundo Silva (2007), quando dietas apresentam teor elevado de fibra em relação aos requerimentos do animal, a relação entre consumo e teor de FDN será negativa. Por outro lado, quando as dietas são pobres em fibra, esta relação será positiva dentro de um mesmo nível de produção. Quando se altera o conteúdo de FDN da dieta pela substituição de grãos por volumosos, a ingestão de MS irá aumentar até que 
não seja limitada pelo enchimento e decresce quando for limitada pelo excesso de produtos metabólicos, demonstrando o efeito de enchimento físico e quimiostático sobre o consumo. No caso de volumosos de baixa qualidade como o bagaço de cana, o efeito de enchimento prevalece, uma vez que o teor de energia deste volumoso é insuficiente para proporcionar um controle quimiostático na regulação da ingestão.

Pelas equações de Madsen et al. (1997) o maior consumo estimado foi o da silagem de milho (independente da taxa de passagem), expresso tanto em kg MS/dia (Tabela 3) como em \% PV (Tabela 4). Cabral et al. (2006) avaliaram o consumo de bovinos mestiços com PV médio de $350 \mathrm{~kg}$, recebendo dietas a base de silagem de milho suplementadas com $10 \%$ de farelo de soja e verificaram consumo de $6,32 \mathrm{~kg} \mathrm{MS} / \mathrm{dia}$, inferior ao obtido neste estudo por meio da equação de Madsen et al. (1997) considerando $k$ de $2 \% / h$; porém, foi semelhante ao estimado pelas equações de Ørskov et al. (1988) e Shem et al. (1995). De acordo com Silva (2007), o consumo é limitado pela demanda de energia e não pelo efeito de enchimento do alimento, quando o teor de FDN estiver abaixo de 50-60\%. Neste estudo, apenas a silagem de milho apresentou teor de FDN inferior a $50 \%(46,28 \%)$, portanto, existe a possibilidade do consumo da silagem não ter sido regulada apenas por mecanismos de enchimento físico, mas também pelo nível de energia. Ainda segundo Silva (2007), nestas situações, a remoção de variações associadas às diferenças entre animais, reduz significativamente a variação nas estimativas e melhora as predições de consumo com base na composição química dos alimentos. Outro fator que pode afetar a ingestão de volumosos fermentados é o teor de umidade. Segundo o NRC (1989), existe uma relação negativa entre a ingestão de matéria seca em dietas com alto teor de umidade (acima de $50 \%$, quando alimentos fermentáveis são incluídos na ração.

De modo geral, as menores estimativas de consumos de MS dos volumosos foram observadas quando utilizou-se as equações propostas por Shem et al. (1995) e Von Keyserlingk \& Mathison (1989). Além disso, no caso 
dos volumosos de baixa qualidade (Feno 90 e bagaço de cana), os valores de consumo estimados pela a equação de Shem et al. (1995) foram muito baixos, inclusive negativo para o bagaço de cana. Soares et al. (2001) avaliando as mesmas equações de predição do consumo de capim Tanzânia sob pastejo por vacas em lactação, verificaram que entre as equações avaliadas, a de Shem et al. (1995) mostrou valores mais próximos da ingestão $(8,0 \mathrm{~kg} \mathrm{MS} / \mathrm{dia})$ estimada pelo método de coleta de fezes com o uso de óxido crômico $(9,6 \mathrm{~kg}$ MS/dia). No entanto, é importante destacar que esta equação foi desenvolvida para forrageiras tropicais, portanto, seu uso pode não se ajustar à predição do consumo de volumosos e subprodutos de qualidade muito inferior, como o Feno cortado aos 90 dias e o bagaço de cana.

Independente da equação utilizada, as estimativas de consumo do Feno 90 e do bagaço de cana foram mais baixas que as obtidas para os demais volumosos avaliados. O menor consumo do feno de baixa qualidade pode também ser decorrente do efeito substitutivo, uma vez que a relação volumoso concentrado de ambas as dietas (Feno 30 e Feno 90) foi a mesma (70:30). Lucci (1997) observou, em vacas em lactação, efeito de substituição de 0,93 e $0,73 \mathrm{~kg}$ de volumoso pelo concentrado. Resultados superiores de consumo encontrados na literatura (Soares et al., 2009) são provavelmente devido a utilização de forragens de melhor qualidade, nos quais a demanda de energia e o valor nutritivo da forragem avaliados foram maiores do que nesse estudo.

Houve diferença $(P<0,05)$ no consumo de MS dos volumosos estimado pelas equações de predição. O consumo do Feno 30, Feno 90, palha de arroz e do bagaço de cana foi semelhante entre as equações de Madsen et al. (1997) e Ørskov et al. (1988), sendo superior às demais equações (tabela 3). A equação de Madsen et al. (1997) permite a detecção de diferenças no consumo quando se compara alimentos de características nutricionais diferentes, pois considera, além das taxas de degradação e de passagem, o enchimento físico ruminal e o consumo de FDN, aumentando a precisão nas estimativas de consumo. 0 consumo obtido a partir desta equação foi estimado considerando as taxas de 
passagem de 2, 3 e 5\%/h. Conforme observado na Tabela 3, a variação nas taxas de passagem alterou $(P<0,05)$ o consumo de $M S$, evidenciando a importância da determinação e inclusão deste parâmetro na estimativa do consumo de volumosos. Segundo Von Keyserlingk \& Mathison (1989), a técnica in situ poderia ser mais eficiente como método para estimar a ingestão voluntária de forragens se as taxas de passagem e degradação fossem determinadas e utilizadas para predizer estes fatores, além de apresentar alta correlação com a ingestão voluntária $(R=0,82)$ quando comparada com a digestibilidade in vivo.

O consumo de MS estimado para o feno de Tifton cortado aos 30 dias foi superior ao feno cortado aos 90 dias (Tabelas 3 e 4), verificado em todas as equações utilizadas. De acordo com Van Soest (1994), o consumo e a digestibilidade das diferentes frações da forragem estão ligados à idade da planta, pois com o avanço no estádio de desenvolvimento, as forrageiras apresentam maiores teores de matéria seca, com baixos teores de proteína e de energia disponíveis e, consequentemente, altos teores de parede celular. Soares et al. (2008), trabalhando com vacas em lactação, determinaram o consumo do capim elefante picado utilizando comedouro automático e compararam com as referidas equações de predição. Os autores observaram que o modelo de Madsen et al. (1997) superestimou o consumo de MS. Porém, quando utilizaram os valores de consumo de FDN e de enchimento ruminal, determinados diretamente no animal, na equação de Madsen et al. (1997), o consumo foi semelhante ao obtido no comedouro automático. Segundo os autores, o modelo de Madsen et al. (1997) seria mais eficiente quando se utiliza valores reais, porém, deve-se considerar a mão-de-obra e o estresse causado no animal. Neste trabalho, o consumo do Feno 30 obtido pelas equações de Ørskov et al. (1988) e de Madsen et al. (1997) apresentaram valores semelhantes e próximos aos encontrados na literatura (Tabela 4).

Exceto para a silagem de milho, os consumos de MS de cada fonte de volumoso obtidos pelo modelo de Ørskov et al. (1988) foram semelhantes aos 
obtidos por Madsen et al. (1997), considerando taxa de passagem de $2 \% / \mathrm{h}$. Berchielli et al. (2001), avaliando o consumo de vacas lactantes em pastagem de capim coast-cross, verificaram que as equações de predição do consumo baseadas nas características de degradação ruminal superestimaram o consumo de MS, entretanto, o modelo proposto por Ørskov et al. (1988) foi o que mais se aproximou ao consumo obtido pelo método de coleta de fezes utilizando o óxido crômico. Apesar de ter sido desenvolvido para volumosos de baixa qualidade (palhadas), o modelo de Ørskov et al. (1988) pode ser utilizado para estimar o consumo de forragens de alta qualidade como o feno 30, pois apresentou valores semelhantes ao encontrado na literatura (Tabela $4)$.

O consumo médio da palha de arroz estimado pelas equações de Madsen et al. (1997) $(\mathrm{k}=2 \% / \mathrm{h})$ e Ørskov et al. (1988) foi de $6,52 \mathrm{~kg} \mathrm{MS} / \mathrm{dia}$, diferindo $(P<0,05)$ das equações propostas por Shem et al. (1995) e Von Keyserlingk \& Mathison (1989) (2,75 kg MS/dia). Mgheni et al. (2001), utilizando a equação de Madsen et al. (1997) $(k=2 \% / h)$ na predição do consumo da palha de arroz em bovinos, verificaram ingestão média de $4,91 \mathrm{~kg}$ de $\mathrm{MS} / \mathrm{dia}$, inferior ao obtido neste trabalho com a mesma equação $(6,59 \mathrm{~kg} \mathrm{MS} / \mathrm{dia})$. Diferenças na técnica de incubação in situ e variações entre animais e alimentos poderão interferir na estimativa do consumo, quando se utiliza estes parâmetros. É importante mencionar que essas equações foram baseadas no potencial de degradação da matéria seca da forragem no rúmen sem considerar a influência exercida pelo manejo dos animais, a forma física em que o alimento foi administrado e o ambiente em que as vacas permaneceram durante o período experimental.

As estimativas de consumo do bagaço de cana foi inferior $(P<0,05)$ em relação às observadas para os demais volumosos, independente da equação utilizada. Pires et al. (2004), trabalhando com novilhas mestiças (Holandês $x$ Indubrasil) alimentadas com bagaço de cana, observaram consumo médio de $1,81 \mathrm{~kg} \mathrm{MS} / \mathrm{dia}$, próximo ao verificado neste estudo quando utilizaou-se a 
equação de Von Keyserlingk \& Mathison (1989). Considerando que o teor de FDN seja negativamente correlacionado ao consumo, quando a repleção ruminal é o fator limitante espera-se o menor consumo para volumosos com maior teor de FDN, como no caso do bagaço de cana ( $85 \%$ FDN).

A variabilidade nos valores de consumo observadas na literatura indica que o teor de FDN pode não ser o principal fator responsável pela regulação do consumo em ruminantes. Forbes (2004) relata que diversos fatores estão relacionados durante o processo de controle da ingestão em ruminantes, como os nutrientes, doenças, condições ambientais e pressão social.

Na tabela 5 são apresentados os coeficientes de correlação $(r)$ entre as equações de predição do consumo e os parâmetros de degradabilidade $a, b$ e $c$ e o enchimento ruminal (ER) para cada um dos volumosos avaliados neste estudo.

A relação entre as estimativas de ingestão de matéria seca e os parâmetros de degradação variou em função do tipo de equação e de volumoso. Entre os parâmetros de degradação utilizados, a taxa de degradação (c) apresentou coeficientes de correlação significativos para todos os volumosos avaliados, sendo altos e positivos com todas equações no caso do Feno30 e só não foi significativa para equação de Shem et al. (1995) no caso da silagem. Entretanto, quando se refere aos volumosos de baixa qualidade (Feno 90, bagaço de cana e palha de arroz), as correlações entre a taxa de degradação e o consumo estimado pelas equações de Shem et al. (1995) e Madsen et al. (1997) não foram significativas. Quanto à equação de Shem et al. (1995), a baixa correlação com a taxa de degradação dos volumosos de baixa qualidade pode ter ocorrido devido ao tipo de forrageira para qual a equação foi desenvolvida, ou seja, pastagens tropicais, que certamente são de melhor qualidade nutricional, comparadas ao bagaço de cana, palha de arroz e feno cortado aos 90 dias. Isto refletiu nos baixos valores de consumo destes volumosos (Tabela 3 ). 
Tabela 5. Coeficientes de correlação $(r)$ entre as equações de predição do consumo e os parâmetros de degradabilidade $a, b$ e $c$ e o enchimento ruminal (ER), observados para cada volumoso.

\begin{tabular}{|c|c|c|c|c|}
\hline Equação & $a$ & $\begin{array}{c}B \\
\text { Silagem de milho }\end{array}$ & $C$ & ER (dias) ${ }^{7}$ \\
\hline V. Keyserlingk ${ }^{3}$ & $-0,51$ & 0,66 & $0,99 *$ & - \\
\hline Ørskov ${ }^{4}$ & $-0,49$ & 0,65 & $0,98 *$ & - \\
\hline Shem $^{5}$ & $0,98 *$ & $-0,54$ & $-0,28$ & - \\
\hline Madsen $^{6}$ & $-0,46$ & $\begin{array}{c}0,64 \\
\text { Feno } 30^{1}\end{array}$ & $0,95 *$ & $-0,99 *$ \\
\hline V. Keyserlingk & 0,19 & 0,55 & $0,99 *$ & - \\
\hline Ørskov & 0,15 & 0,59 & $0,98 *$ & - \\
\hline Shem & 0,47 & 0,32 & $0,95 *$ & - \\
\hline Madsen & 0,06 & $\begin{array}{c}0,61 \\
\text { Feno } 90^{2}\end{array}$ & $0,96 *$ & $-0,99 *$ \\
\hline V. Keyserlingk & 0,43 & 0,07 & $0,89 *$ & - \\
\hline Ørskov & 0,37 & 0,27 & $0,79 * *$ & - \\
\hline Shem & 0,29 & 0,49 & 0,64 & - \\
\hline Madsen & 0,20 & $\begin{array}{c}0,54 \\
\text { Bagaço de cana }\end{array}$ & 0,56 & $-0,99 *$ \\
\hline V. Keyserlingk & 0,05 & $-0,10$ & $0,78 * *$ & - \\
\hline Ørskov & $-0,01$ & 0,30 & 0,46 & - \\
\hline Shem & 0,32 & 0,70 & $-0,35$ & - \\
\hline Madsen & $-0,09$ & $\begin{array}{c}0,24 \\
\text { Palha de arroz }\end{array}$ & 0,26 & $-0,99 *$ \\
\hline V. Keyserlingk & 0,47 & 0,54 & $0,95 *$ & - \\
\hline Ørskov & 0,47 & 0,61 & $0,92 *$ & - \\
\hline Shem & 0,71 & 0,61 & 0,74 & - \\
\hline Madsen & $-0,43$ & 0,69 & 0,38 & $-0,99 *$ \\
\hline
\end{tabular}

${ }^{1}$ Colhido aos 30 dias de rebrote. ${ }^{2}$ Colhido aos 90 dias de rebrote. ${ }^{3}$ Von Keyserlingk \& Mathison (1989). ${ }^{4} Ø$ rskov et al. (1988). ${ }^{5}$ Shem et al. (1995). ${ }^{6}$ Madsen et al. (1997), considerando taxa de passagem de $3 \% / h .{ }^{7} E R=(1-a-b) / k+(b / c+k) / 24 . a=$ fração solúvel, $\mathrm{b}=$ fração potencialmente degradável e $\mathrm{c}=$ taxa de degradação da fração $\mathrm{b}$. ${ }^{*} \mathrm{P}<0,05$ e ${ }^{*} \mathrm{P}<0,06$ pelo Teste de Tukey.

O enchimento físico ruminal (ER), utilizado na equação de Madsen et al. (1997), apresentou correlação alta e negativa $(P<0,05)$ com o consumo voluntário, ou seja, quanto maior o teor de FDN do alimento, maior o enchimento físico, havendo diminuição do consumo. Entretanto, quando se refere à silagem de milho, a correlação do ER com o consumo não se justificaria totalmente, uma vez que outras características poderiam estar prioritariamente limitando sua ingestão, como fatores fisiológicos ou a elevada 
concentração de ácidos orgânicos, decorrentes da fermentação dos seus açúcares solúveis, além dos fatores discutidos anteriormente.

Nakajima \& Nakayama (1997), avaliando o consumo estimado por meio das características de degradação do feno de alfafa, também verificaram alta correlação entre a taxa de degradação e as equações de predição do consumo em ovinos. Segundo os autores, o aumento na acurácia da estimativa melhora significativamente com a inclusão da taxa de passagem e do ER no modelo.

As frações solúvel (a) e potencialmente degradável (b) não foram correlacionados significativamente $(P>0,05)$ com a predição do consumo dos volumosos, considerando as equações utilizadas nesse estudo. Nakajima \& Nakayama (1997) também verificaram pequeno efeito desses parâmetros na acurácia de predição do consumo.

Para a silagem de milho, a correlação da fração b e da taxa de degradação com a equação proposta por Shem et al. (1995) não foi significativa $(P<0,05)$. Conforme mencionado, esta equação foi desenvolvida para forrageiras tropicais e, portanto, na estimativa do consumo de um volumoso fermentado como a silagem, estes parâmetros não se correlacionam com a equação.

Em estudos envolvendo as características de degradação in situ de volumosos, verifica-se o quanto os parâmetros de degradação influenciam a utilização do alimento pelo animal e sua relação com a ingestão. Entretanto, sabe-se que além das frações $a, b$ e c, outras características da forragem podem influenciar a predição da ingestão, como a taxa de redução de partículas maiores, o tempo de colonização destas, assim como a taxa constante de saída de digesta ruminal (Ørskov et al., 1988) e que não estão presentes como variáveis nestas equações.

\section{Conclusão}

Os parâmetros de degradação ruminal têm efeito na estimativa do consumo, variando em função do tipo de volumoso. A taxa de degradação foi o 
MARTINS, A.S. et al. Consumo voluntário de volumosos estimado por meio de parâmetros de degradação ruminal. PUBVET, Londrina, V. 7, N. 15, Ed. 238, Art. 1570, Agosto, 2013.

parâmetro que apresentou maior correlação com as estimativas de consumo. Ao definir um modelo para estimar o consumo de volumosos é importante considerar as características nutricionais, não havendo um único modelo para estimar o consumo de volumosos com qualidades nutricionais diferentes.

\section{Bibliografia}

Agricultural and Food Research Council - AFRC. Technical committee on responses to nutrients: energy and protein requirements of ruminants. 1993. Wallinford: CAB International, 159p.

Agricultural Research Council - ARC. The nutrient requeriments of ruminant livestock. Commonwealth Agricultural Bureaux, London, 1984. 184p.

Berchielli, T.T.; Soares, J.P.G.; Aroeira, L.J.M. Estimativa da ingestão voluntária a partir das características de degradação do capim coast cross (Cynodon dactylon L. Pers.), sob pastejo, por vacas em lactação. Rev. Bras. Zootec., v.30, n.4, p. 1332-1339, 2001.

Berchielli, T.T; Veja, A.G; Reis, R.A. Técnicas de avaliação de consumo em ruminantes: Estado da arte. In: Rennó, F.P.; Silva, L.F.P. (Eds.) Simpósio Internacional Avanços em Técnicas de Pesquisa em Nutrição de Ruminantes, Pirassununga, 2007, Anais... Pirassununga, 2007. p. 305-341.

Cabral, L.S.; Valadares Filho, S.C.; Detmann, E. et al. Consumo e digestibilidade aparente em bovinos alimentados com dietas à base de volumosos tropicais. Rev. Bras. Zootec., v.35, n.6, p.2406-2412. 2006.

Forbes, J.M. The multifactorial nature of food intake control. J. Anim. Sci., v.81, (Suppl.2), p.139-144. 2004.

Forbes, J.M. 1995. Voluntary food intake and diet selection in farm animals. CAB International. Walingford. $532 \mathrm{p}$

Hovell, F.D.B., Ngambi, J.W.W., Barber, W.P., Kyle, D.J. 1986. The voluntary intake of hay by sheep in relation to its degradability in the rumen as measured in nylon bags. Anim.Prod., v.42, n.1, p.111-118.

Lucci, C. S. Nutrição e manejo de bovinos leiteiros. São Paulo: Manole,1997 169p.

Madsen, J., Hvelplund, T., Weisbjerg, M.R. Appropriate methods for evaluation of tropical feeds for ruminants. Anim. Feed Sci. Techn., v.69, n.2, p.53-66, 1997.

Martins, A.S., Zeoula, L.M.; Prado, I.N., Martins, E.N., Loyola, V.R. Degradabilidade ruminal in situ da matéria seca e proteína bruta das silagens de milho e sorgo e de alguns alimentos concentrados. Rev. Bras. Zootec., Viçosa, v. 28, n. 4, p. 1109-1117, 1999.

Mehrez, A.Z., Ørskov, E.R. 1977. A study of the artificial fiber bag technique for determining the digestibility of feeds in the rumen. J. Agric. Sci., 88(3):645-665.

Mertens, D.R. Predicting intake and digestibility using mathematical models of ruminal function. J. Anim. Sci., 64(8):1548-1558. 1987.

Mertens, D.R. Regulation of forage intake. In: Forage Quality, Evaluation, and Utilization, 1994, Wisconsin. Proceedings... Wisconsin: 1994. p.450-493.

Mgheni, D.M.; Ndemanisho, E.E.; Hvelplund, T., Weisbjerg, M.R. Evaluation of the feeding value of two tropical cereal straws, maize stover, rice straw and their botanical fractions by nylon and mobile bag technique. African J. Sci. Technol., v.2, n.1, p. 65-71, 2001. 
Moore, J. E. Forage Crops In: Hoveland, C. S. (Ed.). Crop quality, storage, and utilization.. Madison: American Society, Inc., and Crop Science Society of America, Inc. 1980. p.61-91.

Nakashima, Y.; Nakayama, I. Prediction of voluntary intake, apparent digestibility dry matter intake of hays by sheep from rumen degradation characteristics, ruminal outflow rate and rumen fill. Grasslands Sci., v.42, n.4, p. 283-288, 1997.

National Research Council - NRC. Nutrient requeriments of dairy cattle. 7 ed. Washington, D.C.: National Academy Press, 2001. 381 p.

National Research Council - NRC. 1989. Nutrient requirements of dairy cattle. 6.ed. Washington D.C.: National Academy Press. 420p.

Mendes Neto, J.; Campos, J.M.S.; Valadares Filho, S.C.; Lana, R.P.; Queiroz, A.C.; Euclydes, R.F. Consumo, digestibilidade, desempenho, desenvolvimento ponderal e economicidade de dietas com polpa cítrica em substituição ao feno de capim Tifton-85 para novilhas leiteiras. Rev. Bras. Zootec., v.36, n.3, p. 626-634, 2007.

Nussio, L.G.; Manzano, R.P.; Pedreira, C.G.S. Valor alimentício em plantas do gênero Cynodon. In: Peixoto, A.M., Moura, J.C., Faria, V.P. (Eds) Manejo de pastagem de tifton, coastcross e estrela, 15., 1998, Piracicaba. Anais... Piracicaba: FEALQ, p.203-239, 1998.

Ørskov, E.R., Reid, G.W., Kay, M. 1988. Predicting of intake by cattle from degradation characteristics of roughages. Anim. Prod., 46(1):29-34.

$\varnothing$ rskov, E.R., McDonald, I. 1979. The estimation of protein degradability in the rumen from incubation measurements weighted according to rate of passage. J. Agric. Sci., 92(1):499-503.

Pires, A.J.V.; Valadares Filho, S.C.; Pereira, O.G.; Cecon, R.C.; Silva, F.F.; Silva, P.A.; Veloso, C.M. Novilhas alimentadas com bagaço de cana-de-açúcar tratado com amônia anidra e ou sulfeto de sódio. Rev. Bras. Zootec., v.33, n.4, p. 1078-1085. 2004.

Reis, R.A.; Rodrigues, L.R.A. Amonização de volumosos. FUNEP: Jaboticabal, Universidade Estadual Paulista, 1993. 22p.

SAS INSTITUTE. SAS user's guide: Statistics, 4a ed. Version 8.1, v. 2. Cary: SAS Institute, 943 p., 2000.

Shem, M.N., Ørskov, E.R., Kimambo, A.E. 1995. Prediction of voluntary dry-matter intake, digestible dry matter intake and growth rate of cattle from degradation characteristics. $J$. Anim. Sci., 60(1):65-74.

Silva, D.J.; Queiroz, A.C. Análise de alimentos - métodos químicos e biológicos. 3ed., Viçosa: UFV, 2002. 235p.

Soares, J.P.G.; Salman, A.K.D.; Berchielli, T.T.; Aroeira, L.J. M.; Verneque, R.S. Predição do consumo voluntário do capim Tanzânia (Panicum maximum, J. cv. Tanzânia), sob pastejo, por vacas em lactação a partir das características de degradação. Rev. Bras. Zootec., v.30, (supl. 6), p. 2176-2182, 2001.

Soares, J.P.G., Berchielli, T.T.; Salman, A.K.D.; Deresz, F.; Oliveira, A.D.;Verneque, R.S. Comparações entre equações de predição de consumo de capim elefante cortado para vacas lactantes. Arch. Zootec., v. 57 (218), p.235-246, 2008.

Soares, J. P. G. ; Deresz, F. ; Aroeira, L.J.M. ; Salman, A.K.D.; Efeito da suplementação de concentrado sobre o consumo de capim-elefante picado, por vacas mestiças - Porto Velho, RO: Embrapa Rondônia, 2009.22 p. - (Boletim de Pesquisa e Desenvolvimento / Embrapa Rondonia, 1677-8618; 60).

Valadares Filho, S.C. Utilização da técnica in situ para avaliação dos alimentos. In: Simpósio Internacional de Produção de Ruminantes, 1994. Maringá. Anais... Maringá: UEM. p. 95-118. 
Van Soest, P.J. Nutritional ecology of the ruminant. 2ed. Ithaca: Comstock Publication Association, 1994. 476p.

Von Keyserlingk, M.A.G., Mathison, G.W. 1989. Use of the in situ technique and passage rate constants in predicting voluntary intake and apparent digestibility of forages by steers. Can. J. Anim. Sci., 69(5):973-987. 